\title{
Two-Step Continuous Cooling Heat Treatment Applied in a Low Carbon Bainitic Steel
}

\author{
Pedro José de Castro ${ }^{a *}$ (D), Antonio Carlos de Figueiredo Silveira ${ }^{a}$ (D), Thiago Marques Ivaniski ${ }^{a}$ (D), \\ Cristiano José Turra ${ }^{(\mathbb{D}, \text { Jeremy Epp }}{ }^{b, c}$ Alexandre da Silva Rocha ${ }^{a}$ (D) \\ ${ }^{a}$ Universidade Federal do Rio Grande do Sul, Departamento de Metalurgia, Av. Bento Gonçalves, \\ 91509-900, Porto Alegre, RS, Brasil \\ ${ }^{b}$ Leibniz-Institut für Werkstofforientierte Technologien - IWT, Badgasteiner Str., 28359, Bremen, \\ Germany \\ ${ }^{c}$ University of Bremen, MAPEX Centre for Materials and Processes, Bremen, Germany
}

Received: July 24, 2020; Revised: December 5, 2020; Accepted: January 23, 2021

\begin{abstract}
Thermo-mechanical treatments using continuous cooling after forging are an established method for producing bainitic steels, mainly because of the elimination of energy intensive additional heat treatment processes. The cooling is usually employed in an uncontrolled manner in the industrial sector, which can be detrimental to the resulting microstructural morphology and, consequently, to the final product properties. In this study, a new controlled two-step cooling route based on the principles of bainitic displacive growth was designed and applied in a $0.18 \mathrm{C}$ (wt-\%) steel. Inverse finite element method was used on the cooling data to obtain the evolution of temperatures for the samples during cooling, allowing to assess point to point cooling rates. Investigations via X-ray diffraction, optical microscopy analysis and hardness testing revealed a variation of bainitic morphology, namely, the transition from granular bainite to lath-like bainite with relatively high hardness and constituents/ phase boundaries than the pre-treated microstructure.
\end{abstract}

Keywords: low carbon bainitic steel, heat treatment, granular bainite, lath-like bainite.

\section{Introduction}

Recently, there has been an increasing economic and ecological interest in the reduction of energy consumption. In the steel production and manufacturing industry, aiming to make the processing chain leaner by replacing energy intensive production steels, new alloys and processing routes are being employed. One expressive example of this is the substitution of quenched and tempered steels by continuously cooling steels, which achieve their final microstructure right after manufacturing integrated thermomechanical processing (like hot rolling or forging) ${ }^{1-3}$. This change in the production chain results in a microstructural transition from tempered martensite to a wide array of bainitic microstructures in which the morphology of the microstructure and the resulting mechanical properties are closely related to the alloy's chemical composition. Bainite transformation is a physical phenomenon extensively studied in the field of modern metallurgy ${ }^{4-6}$. This interest can be attributed to the difficulties regarding the understanding of the transformation's thermodynamical aspects $^{7}$ as well as the wide range of industrial applications this microstructure provides ${ }^{8-10}$.

In view of exploring the thermodynamical aspects of the bainitic transformation, multi-step heat treatments (MSHT) have been developed in the last years. They consist of adding temperature steps to the austempering process. This group of heat treatments (HT), which are focused on further

*e-mail: pjdcastro73@gmail.com enhancing the outcome of conventional one-step austempering treatments, has been proven to be an effective tool for fine tailoring the properties of bainitic laths and, especially, the characteristics of resulting room temperature retained austenite (RA). Wang et al. ${ }^{11}$, utilized a MSHT to achieve nanostructured bainite on a medium $(0.30 \%) \mathrm{C}$ steel. This was achieved due to carbon partitioning to the austenite, which reduced the martensite start temperature of the steel with each step, thus allowing succeeding bainite formation to have continuously thinner laths. The succeeding steps also allowed the transformation of blocky RA into bainite and film austenite, increasing both ductility and yield strength of the multi-step treated samples. Avishan et al. ${ }^{12}$, investigated the effect of a two-step HT with focus on optimization of the transformation induced plasticity (TRIP) effect through tailoring the properties of the retained austenite. They found out that extensively refining the retained austenite films results in an overly stable austenite, hindering the benefit of the TRIP effect. Mousalou et al. ${ }^{13}$, also achieved nanostructured bainite in a low carbon $(0.26 \%) \mathrm{C}$ steel. They reported expressive toughness improvement in comparison to conventional one-step treatment, which was attributed to the insertion of a higher variety of variants, increasing the high angle boundaries and thus, hindering crack propagation. Li et al. ${ }^{14}$, modified the conventional two-step HT by adding a brief step before beginning a lower temperature austempering. With the first step being at a higher temperature, they lowered the number of sites for bainite nucleation as well as the 
undercooling between the $1^{\text {st }}$ and $2^{\text {nd }}$ steps, resulting in higher amounts of RA by sacrificing bainitic lath refinement. With this, they achieved a more effective TRIP effect, resulting in improved elongation and ultimate tensile strength. These studies showed the benefits of conducting multiple step austempering. However, no efforts have been directed to designing multiple step continuous cooling heat treatments.

Accordingly, the present work addressed the microstructural response of a low carbon bainitic steel, DIN 18MnCrSiMo6-4, when subjected to a two-step continuous cooling after austenitizing, simulating the hot forging step. A usual treatment for this material is an uncontrolled air cooling directly after hot rolling or forging operations. The air-cooled microstructure consists of a ferritic bainitic matrix embedded by quasi axial islands of martensite-austenite constituents (MA), namely, Granular Bainite (GB) that characterizes the as-received microstructure of this material. The focus of the present work was to evaluate the effects of dividing the cooling process into two steps with different cooling rates. The first step involves intense cooling and the second air-cooling. The aim of the first step is to provide a higher driving force for the beginning of the bainitic transformation, influencing the resulting bainitic morphology and its properties.

\section{Methodology}

In order to analyze the effect of cooling strategy on the microstructure, optical microscopy, hardness testing and $\mathrm{X}$-ray diffraction were utilized. Furthermore, simulation of the cooling curves to assess the temperature map evolution was performed. Accordingly, this section describes all utilized materials, methods and techniques, including sample description and preparation.

\subsection{Samples geometry, chemical composition and initial microstructure}

Samples were machined into cylinders of $54 \mathrm{~mm}$ length per $38 \mathrm{~mm}$ diameter from DIN 18MnCrSiMo6-4 hot rolled bars from Swisstec (SwissSteel), Emmenbrücke, Switzerland. Table 1 presents the chemical composition of the DIN $18 \mathrm{MnCrSiMo6}-4$ steel employed in this investigation.

The as-received microstructure of the samples is presented and further discussed in the results section 3.1.

\subsection{Microstructural characterization}

All samples were cut in their half-length, for analysis of the transverse section and then ground by a sequence of silicon carbide papers until 1200 grit. Sequentially, samples were polished with $1 \mu \mathrm{m}$ diamond paste for metallographic analysis.

For optical microstructure analysis, samples were etched with $2 \%$ vol. Nital etchant for 10 seconds whereas for the analysis of prior austenitic grain size (PAGS), the samples were etched with an aqueous solution of picric acid ( $42 \mathrm{~mL}$ wetting agent, $58 \mathrm{~mL}$ of distilled water and $2.5 \mathrm{~g}$ of picric acid) by swabbing during 6 minutes. Micrographs of the transversal section's core, middle radius and surface regions of the heat-treated samples were analyzed. For the as-received condition, only the mid-radius was analyzed as a reference condition. The Vickers hardness was also investigated in the aforementioned regions. For each region 5 indentations were carried out with a load of $1 \mathrm{kgf}$ and an application time of 10 seconds.

$\mathrm{X}$-Ray diffraction (XRD) analysis was also carried out in the same regions as in the hardness and metallography analysis. A diffractometer Seifert MZ VI E, GE Inspection technologies operated at $33 \mathrm{kV}$ and $40 \mathrm{~mA}$ with a $\mathrm{Cr}$ radiation source (wavelength of $\lambda=2,29 \AA$ ) was used in the analysis. The diffraction intensities were acquired by a line position sensitive detector in the range of $60^{\circ}<\theta<164^{\circ}$ with a total scanning time of $1.3 \mathrm{~h}$. In order to avoid the influence of residual stresses generated by the sample preparation, electrolytic removal of a $100 \mu \mathrm{m}$ layer was performed by a solution of $20 \% \mathrm{H}_{3} \mathrm{PO}_{4}$ and $80 \% \mathrm{H}_{2} \mathrm{SO}_{4}$ before XRD measurements. The obtained diffraction patterns were analyzed by Rietveld Refinement method with the software TOPAS 4.2 (Bruker-AXS, Karlsruhe, Germany). As the quantity of carbides in this steel is low and thus of limited detection with this method, only $\alpha$-Fe (bainitic ferrite/ martensite) and austenite were considered in the Rietveld Refinement. The instrumental contribution to the peak broadening was removed by convolution of instrumental function determined with NIST $\mathrm{LaB}_{6}$ standard calibration powder. For the calculation of the austenite carbon content, the Dyson and Holmes equation was used ${ }^{15}$.

\subsection{Development of the aimed two-step continuous cooling}

Aiming to achieve the lowest possible temperature for bainitic transformation, while avoiding austenite transformation to martensite or polygonal ferrite, a cooling path was designed, as shown in Figure 1, by the dashed line curve superposed to the utilized steel's continuous cooling transformation diagram (CCT). This cooling path consists of employing two different cooling media, the first generating a high cooling rate and the second a lower cooling rate, as indicated by the dashed line in Figure 1. The aim was to reach $450{ }^{\circ} \mathrm{C}$ at the beginning of the bainitic transformation and then transition to a slow cooling across the bainitic field.

It should be noted that utilizing a one-step CCT diagram for a two-step heat treatment will result in deviations in the transformation curve shape because of the different evolution in length change caused by the cooling rate variation in the proposed heat treatment. However, the deviations in this work should be minor, as the beginning of transformations should only be delayed by the high cooling rate of the first step ${ }^{16}$.

Based on the elaborated thermal route, a preliminary investigation was done to test room temperature $\left(20^{\circ} \mathrm{C}\right)$ oil and water baths as cooling media for the first step. The quenching data allowed estimating the required time for

Table 1. Chemical composition of $18 \mathrm{MnCrSiMo6}-4$ steel, in mass percentage.

\begin{tabular}{ccccccccccccc}
\hline Element & $\mathrm{C}$ & $\mathrm{Si}$ & $\mathrm{Mn}$ & $\mathrm{S}$ & $\mathrm{Ni}$ & $\mathrm{Cr}$ & $\mathrm{Cu}$ & $\mathrm{Mo}$ & $\mathrm{Al}$ & $\mathrm{Ti}$ & $\mathrm{N}$ & $\mathrm{Fe}$ \\
\hline $\mathrm{Ma}-\%$ & 0.18 & 1.19 & 1.42 & 0.015 & 0.063 & 1.17 & 0.10 & 0.27 & 0.005 & 0.004 & 0.01 & $\mathrm{Bal}$ \\
\hline
\end{tabular}




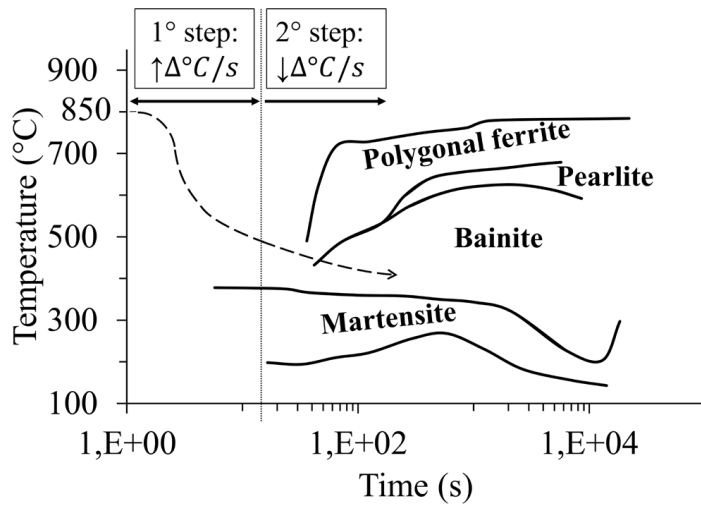

Figure 1. Cooling path plotted over the steel's continuous cooling transformation diagram. The vertical dashed line indicates the transition between the two steps.

achieving the goal temperature for each cooling medium and the respective cooling rate, and thus the first step time. Subsequently, the two-step heat treatment was carried out based on the obtained data about the higher intensity medium followed by air. The chosen temperature for austenitizing was $1000^{\circ} \mathrm{C}$ based on the works of Silveira et al. ${ }^{17}$. With this austenitization temperature, the PAGS of the as-received and post-treatment conditions remained similar, ruling out the influence of the Hall-Petch strengthening on the hardness comparison. All of the heat treatment steps were carried out at atmospheric pressure, with aid of a muffle furnace with standard air atmosphere.

In order to evaluate the cooling rates and the reproducibility of the proposed thermal routes, thermocouples were inserted into the samples. Furthermore, the measured cooling curves were used for the determination of heat transfer coefficients, by means of Finite Element Method (FEM) inverse analysis. The simulation allowed evaluating the temperature field evolution, and thus, the calculation of the cooling rates at any samples' positions.

Temperature mapping was determined by means of 3 thermocouples distributed along the sample's height, as illustrated in Figure 2. For the assessment of reproducibility, each heat treatment was carried out three times.

\subsection{Simulation of two step continuous cooling treatment by finite element method}

By means of the experimental cooling curves data, an inverse analysis was done in order to evaluate the input parameters for the simulation of the two-step continuous cooling heat treatment. FEM was performed by DEFORM ${ }^{\circledR}$ commercial software package. The simulation model considered the sample as a physical model based on the constitutive heat exchange equations ${ }^{18-20}$. Thereby the evolution of the temperature in the system is given by:

$\rho C \frac{\partial T}{\partial t}=\frac{\partial}{\partial x}\left(k \frac{\partial T}{\partial x}\right)+\frac{\partial}{\partial y}\left(k \frac{\partial T}{\partial y}\right)+\frac{\partial}{\partial z}\left(k \frac{\partial T}{\partial z}\right)+Q$

Where $T(x, y, z, t)$ is the transient temperature distribution in ${ }^{\circ} \mathrm{C}, \rho$ is the material density, $C$ is the specific heat, $k$ is the thermal conductivity and $\mathrm{Q}$ is the internal heat generation per volume unit. Equation 1 describes the evolution of temperature through time and is governed by the competition of internal heat conduction and dissipation. The boundary conditions considered were those of radiation, conduction and convection, which are modelled by the following equations:

$$
\begin{aligned}
& \theta_{r}=\sigma_{s} \cdot \varepsilon\left(T^{4}-T_{0}^{4}\right) \\
& \theta_{c}=h\left(T-T_{0}\right)
\end{aligned}
$$

Where $\theta_{r}$ is the radiative heat flux, $\theta_{c}$ is the convective heat flux, $\sigma_{s}$ is the Stefan-Boltzmann constant, $T_{0}$ is the room temperature, $\varepsilon$ is the emissivity and $T$ is the sample temperature. The $h$ value represents the global conduction and convection heat transfer coefficients, which vary with the thermal conductivity $k$ and temperature. The heattreatment modelling consisted of dividing the cooling into multiple simulation steps depending on global heat transfer coefficient, thermal conductivity and specific heat, according to the values of these parameters for the specific chosen step. In this regard, this heat treatment simulation considered its input parameters based on the following three steps:

1. high cooling rate of the first step;

2. a transitional period from the first to the second step;

3. calm air cooling in the second step.

The second simulation step was implemented in order to account for the smooth transitional behavior of cooling curves subjected to transition in cooling media.

For each region of the sample (top, core and bottom), four points were considered to more accurately evaluate the temperature evolution with the FEM: one shared the center position of the thermocouple and three more were distributed in a radius of $0.5 \mathrm{~mm}$ around the thermocouple center. A tetrahedral mesh type was used for modelling the sample and the boundary conditions were based on the chosen heat treatment parameters. Table 2 presents the simulation parameters.

\section{Results}

This section will address the results of the present work with brief discussion. A general in-depth discussion is carried out in section 4 .

\subsection{Characterization of as-received condition}

Regarding microstructural characterization, indications were done accompanying all presented micrographs. In this manner, lath-like bainite (LLB) was indicated with parallel arrows, indicating its growth directions. Ellipses were used to delimitate regions with granular bainite (GB), polygonal ferrite (PF) and martensite-austenite constituents (MA). Grain boundary superposed dashed lines indicated prior austenite grain boundary (PAGB) locations.

Figure 3 a shows the microstructure and hardness results, whereas Figure $3 \mathrm{~b}$ the austenitic grain and austenitic grain size for the as-received condition. Figure $3 \mathrm{c}$ shows the area indicated by a rectangle in Figure 3a in a higher magnification. Additionally, in Figure 3c, the scale (right lower corner) shows the numeric value of the microstructure corresponding prior austenitic grain size. 

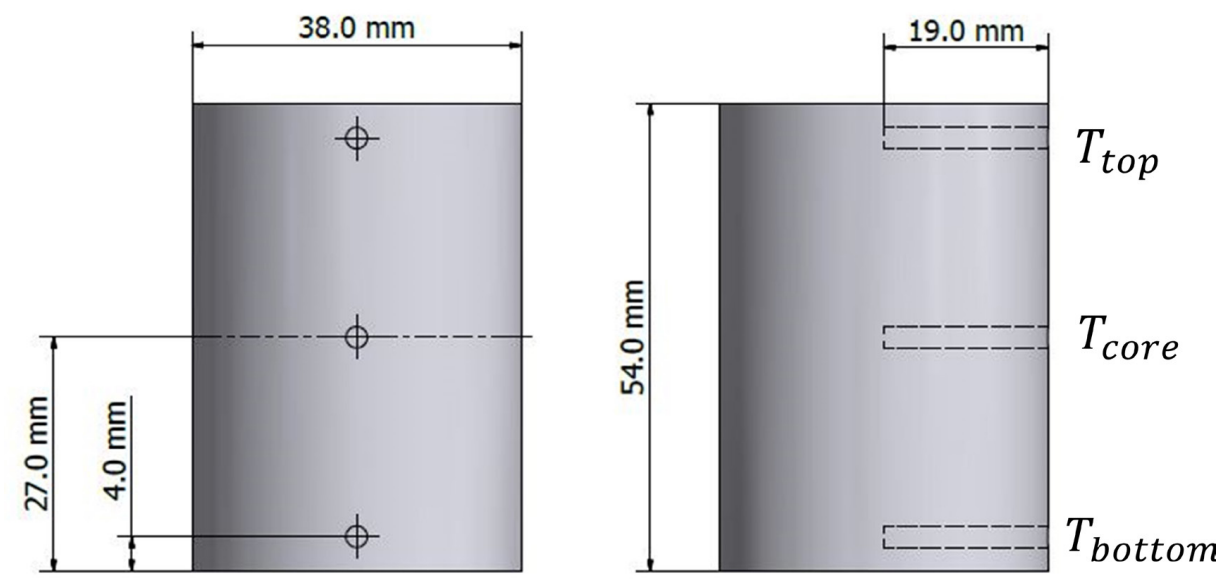

Figure 2. Localization of inserted thermocouples in the sample. (a) frontal view and (b) lateral view. T means thermocouple and the subscripts its positions.

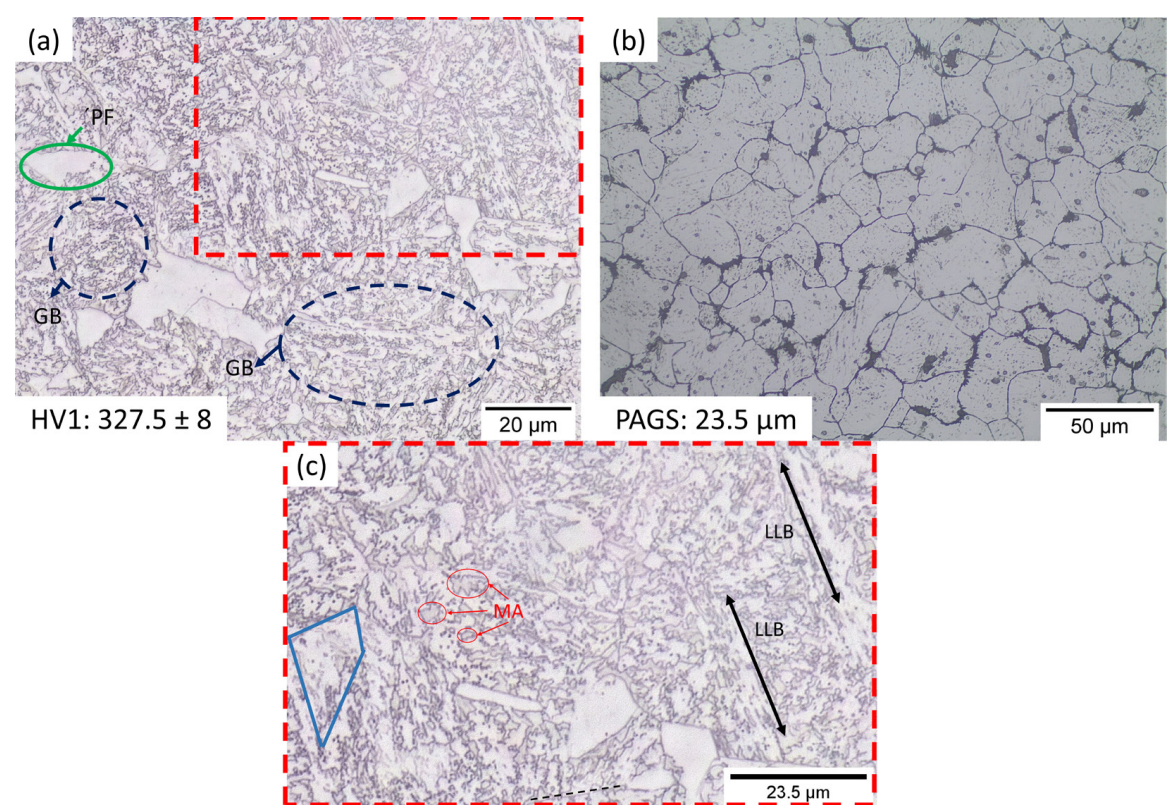

Figure 3. Micrographs of the as-received material: (a) bainitic microstructure (b) austenitic grains and (c) magnified rectangle of (a).

Table 2. Parameters used in the simulation.

\begin{tabular}{lll}
\hline Austenitization temperature $\left({ }^{\circ} \mathbf{C}\right)$ & 1000 & \\
\hline Room Temperature $\left({ }^{\circ} \mathbf{C}\right)$ & 20 & Elements \\
\hline Emissivity $(-)$ & $0,8-0,9$ & 28477 \\
\hline \multirow{2}{*}{ Number of nodes and elements } & Nodes & \\
\cline { 2 - 3 } & 6521 & \\
\hline Type of Mesh & Tetragonal & \\
\hline Solver & Newthon-Raphson & \\
\hline
\end{tabular}

The as-received condition of the material presents a granular bainitic matrix with MA constituents, PF and scarce regions of lath-like bainite, as shown in Figure 3a. Figure 3b shows this microstructure's characteristic PAGB in which the grain size was measured. Figure $3 \mathrm{c}$ shows the selected rectangular area of Figure $3 \mathrm{a}$ with higher magnification. In this image it is possible to identify the MA constituents, indicated by the ellipses. Parallel arrows indicated the bainite lath and its growth direction and the dashed line indicated a grain boundary.

This microstructure is typically obtained in low carbon, medium silicon steels air cooled ${ }^{21,22}$, which, in this case, was $1{ }^{\circ} \mathrm{C} / \mathrm{s}$. The lack of cementite in this microstructure is due to the steel's silicon content, which has low solubility 
in cementite, delaying its precipitation considerably ${ }^{23}$. As shown by an area delimited by a trapezoid shape in Figure 3c, some regions exhibit higher resistance to Nital etching. Reisinger et al. ${ }^{24}$, confirmed in their work that these regions are indeed also granular bainite, however, they have a preferred crystallographic orientation of $\{001\}$ for the bainitic growth direction.

\subsection{Two-step continuous cooling heat treatment}

Figure 4 shows the resulting preliminary oil and water cooling as well as the curve for the conventional air cooling (approximately $1^{\circ} \mathrm{C} / \mathrm{s}$ ) plotted over the CCT diagram. Figure 4 only presents the obtained data for the bottom thermocouple, as this region was the one with highest reached cooling rate. Because there was only interest in evaluating the cooling rate for the first treatment step, further cooling data was omitted in Figure 4.

As Figure 4 indicates, the oil medium would not avoid the polygonal ferrite field and only water would satisfy the high cooling rate prerequisite for the two-step heat treatment in development. Therefore, $20^{\circ} \mathrm{C}$ water was chosen as cooling medium for the first step. The data from the preliminary quenching experiments allowed then estimating the required time in the water bath for the first step of the heat treatment.

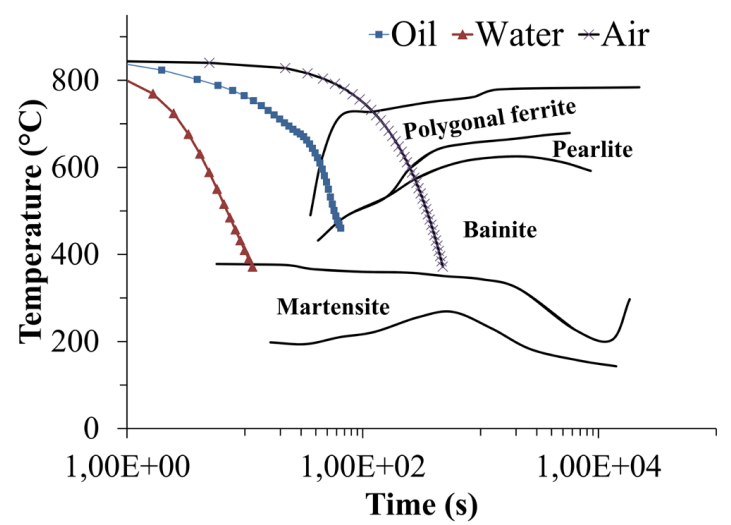

Figure 4. Cooling curves obtained from water, oil and air plotted over the studied material's CCT.
By comparing the overall surface finish of the twostep samples with the direct quenched ones through visual inspection, there were no apparent differences. This is expected, since the proposed heat treatment can be seen as an interrupted quenching treatment.

Figure 5a shows the average temperature of all cooling experiment trials, as well as two vertical dashed lines at 11 and 21 seconds. The $11 \mathrm{~s}$ dashed line accounts for the end of the first heat treatment step, it means, when the sample was removed from the water and air cooling starts (second step). The $21 \mathrm{~s}$ dashed line indicates the lowest temperature reached before the outer region's temperature began to rise due to heat exchange with the core, already in air. The average process cooling rates were calculated based on the highest process temperature (austenitization) for these two intervals, these results are shown in Figure 5b. Further in section 3.3, experimental and simulation results are compared for the $11 \mathrm{~s}$ and 21s instants indicated in Figure 5a.

Based on the superposition to the CCT curve of the material, the use of water in the first step of the two-step heat treatment and air for the second step would be enough to begin the bainitic transformation in lower temperatures and the air cooling would enable avoiding the transformation from austenite to polygonal ferrite and martensite. The curves shown in Figure 5a exhibited a transitional cooling behavior between the end of the first step and the lowest temperature reached, also accompanied by a high dispersion in the obtained data. However, as shown in Figure $5 \mathrm{~b}$ as the heat treatment progressed in air, the cooling rates tended to a uniform value, around $20^{\circ} \mathrm{C} / \mathrm{s}$. This trend is also seen in the dispersion of the cooling rates, which lowered along with the continuity of the treatment.

The temperature evolution indicates that, after reaching a maximum gradient, an inversion of temperature occurs between the core and outer surfaces, implying that a progress towards homogeneity is occurring before any transformation takes place. From a stress evolution perspective, this is desirable, since the transformations will occur practically simultaneously with a low temperature gradient during continuous cooling, similarly as in martempering ${ }^{25}$. Furthermore, even for thicker parts where the transformations occur with a higher (a)

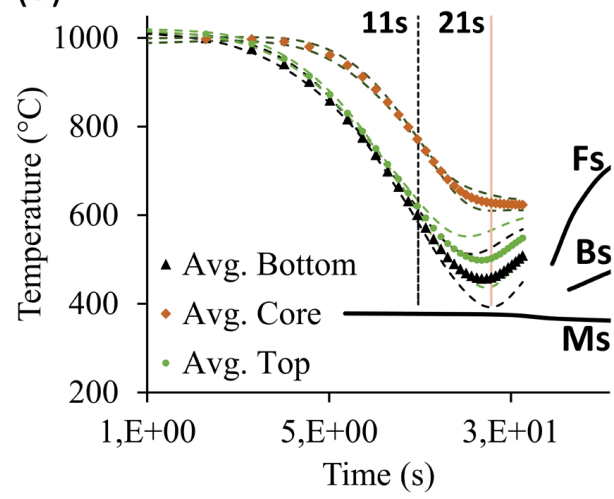

(b)

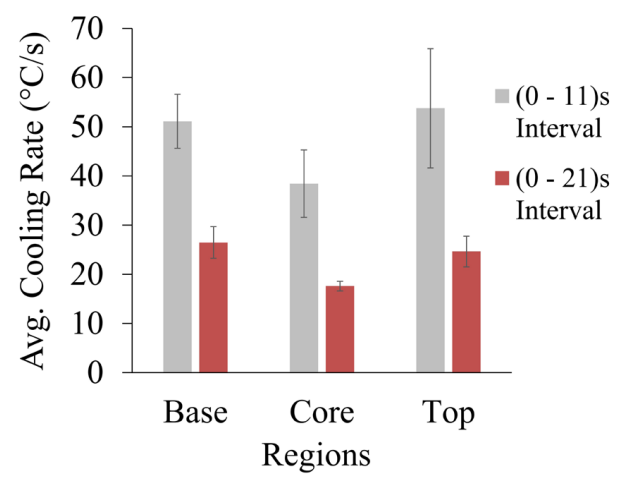

Figure 5. (a) Average cooling curves. The dashed lines accompanying the curves indicate the range of temperature variations. (b) Measured cooling rates for the experimental condition for each region from the beginning to the end of the first step (at $11 \mathrm{~s}$ ) and to the minimum temperature $(21 \mathrm{~s})$. 
temperature gradient, the volumetric change from austenite to bainite is not as pronounced as for martensite ${ }^{26}$. Therefore, considerable residual stresses gradients are not expected as in a conventional quenching process. Some early surface residual stress measurements showed an axisymmetric compressive residual stress at about $200 \mathrm{MPa}$ in the surface.

\subsection{Two-step continuous cooling simulation}

Figure 6 presents the results obtained by the inverse FEM analysis using the obtained experimental data for the heat transfer coefficients: global heat transfer coefficient $h$, heat conductivity $k$ and specific heat $c$, which were the main input values used in the simulation model.

The simulation results are shown in Figure 7. Figure 7a shows the simulated cooling curves plotted over the experimental data and Figure $7 \mathrm{~b}$ and $\mathrm{c}$ the temperature maps at 11 and 21 seconds, respectively.

Figure 8 shows the cooling rates for the experimental and simulated conditions at 21 seconds, determined by inverse FEM analysis from the data shown in Figure 7.

Although the used model for the treatment did not reproduce the temperature rise during the third simulation step, as seen in Figure 7a, the overall experimental and simulated cooling rates at the end of the second step were similar, as shown in Figure 8. Therefore, the results obtained from the temperature maps at 21 seconds, which correspond to the approximate cooling rates before the beginning of bainitic transformation, were extrapolated for the regions in which the microstructural observations were made, allowing a comparison between the obtained cooling rate and resulting post-treatment microstructural morphology. The experimental and simulated cooling rates will be presented along with microstructures in the next section.

\subsection{Microstructural characterization of the two- step heat treatment}

Figure 9a shows the average previous austenitic grain size for the $1000{ }^{\circ} \mathrm{C}$ austenitization after 20 minutes holding time. Figure $9 \mathrm{~b}$ and $\mathrm{c}$ show the microstructure and hardness for the core region of the sample, which was subjected to an average cooling rate of $27^{\circ} \mathrm{C} / \mathrm{s}$ in the 0 to 21 seconds interval. Figure 9c shows some PAGB superposed dashed lines and arrows parallel to direction of bainitic laths growth direction. Here, the scale was also modified to show the respective numerical value of the corresponding microstructure PAGS.

As seen in Figure 9a abnormal grain growth resulting from the total or partial dissolution of precipitates such as TiC and AlN occurred. This phenomenon causes undesirable microstructural inhomogeneity because of its anisotropic properties. Regarding the bainitic transformation, Lan et al..$^{27}$, compared the effect of PAGS on the bainitic transformation in view of the distribution of carbon in the lattice, concluding that coarser austenitic grains contribute to the formation of interlocked bainitic laths and increases the temperature for beginning of bainitic transformation. Furthermore, Caballero et al. ${ }^{28}$, concluded that larger PAGS favors the formation of granular bainite, which in turn favors the formation

\begin{tabular}{|c|c|c|c|}
\hline & $\begin{array}{l}1 \text { - High cooling } \\
\text { rate on liquid } \\
\text { bath. }\end{array}$ & $\begin{array}{c}2 \text { - Transition } \\
\text { between cooling } \\
\text { media }\end{array}$ & $\begin{array}{l}2 \text { - Low cooling } \\
\text { rate on air. }\end{array}$ \\
\hline$h$ & $8.5 W \cdot m^{2} / K$ & $8 W \cdot m^{2} / K$ & $0.02 W \cdot m^{2} / K$ \\
\hline$k$ & $50 \mathrm{~W} / \mathrm{m} \cdot \mathrm{K}$ & $25 \mathrm{~W} / \mathrm{m} \cdot \mathrm{K}$ & $35.5 \mathrm{~W} / \mathrm{m} \cdot \mathrm{K}$ \\
\hline$c$ & $1.2 \mathrm{~J} / \mathrm{K} \cdot \mathrm{kg}$ & $1.2 \mathrm{~J} / \mathrm{K} \cdot \mathrm{kg}$ & $4 \mathrm{~J} / \mathrm{K} \cdot \mathrm{kg}$ \\
\hline
\end{tabular}

Figure 6. Each step considered for the simulation with the respective heat exchange parameters.

(a)

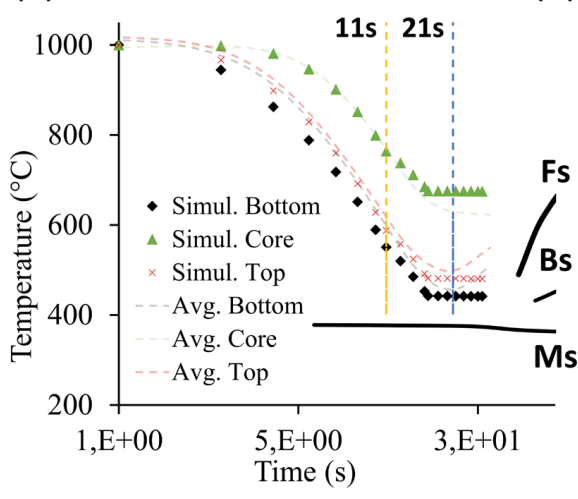

(b)

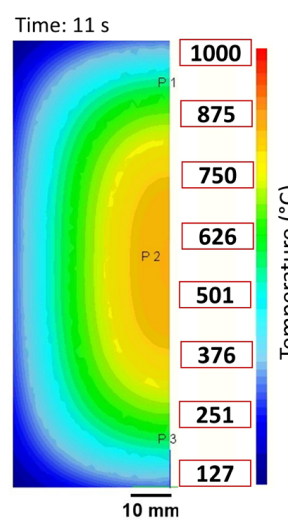

(c)

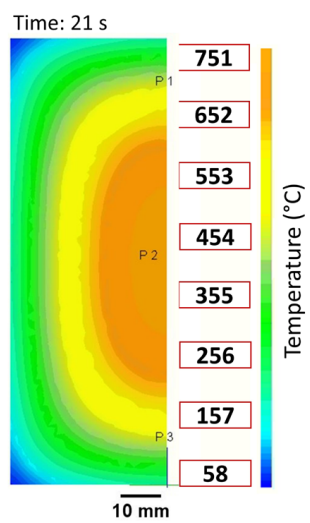

Figure 7. Simulation of the two-step continuous cooling. (a) comparison of simulated and experimentally (dashed lines) measured cooling curves. (b and c) show the temperature maps and position of thermocouples (P1, P2 and P3) at (b) 11 seconds and (c) 21 seconds. 
of mixed granular and lath-like bainite microstructures for steels with inhomogeneous grain size.

Figure 10a shows the microstructure and hardness for middle radius of the same sample. This region had a simulation obtained average cooling rate of $31^{\circ} \mathrm{C} / \mathrm{s}$ in the interval between 0 to 21 seconds. Figure $10 \mathrm{~b}$ shows the magnified delimited region of (a) and indications of a region of GB with an ellipse, PAGB with superposed dashed lines and arrows parallel to bainitic lath growth direction. The scale was modified in order to show the numerical value of the microstructure corresponding PAGS.

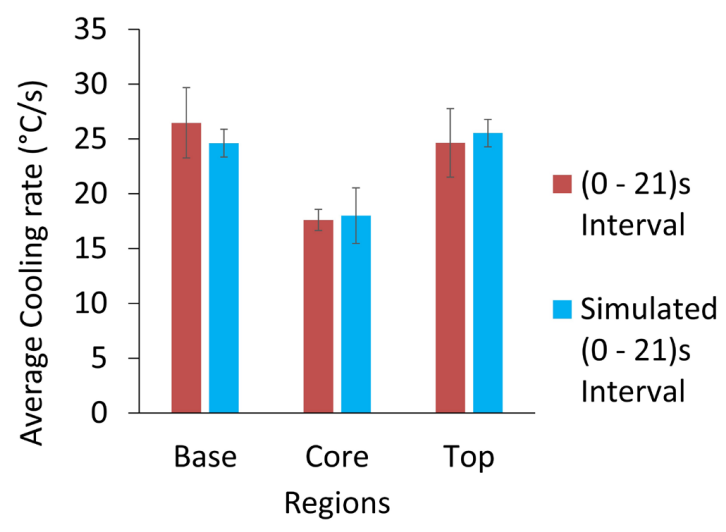

Figure 8. Experimental and simulated cooling rates for each region from the heat treatment beginning until 21 seconds.

\subsection{Phase quantification through X-Ray diffraction}

Table 3 presents the austenite and ferrite proportions obtained by X-ray diffraction. As seen in the table, there is a slight increase in the ferrite quantity and austenite carbon content for the two-step heat-treated sample. Additionally, the ferrite volume can be considered to be only comprised of bainitic ferrite since the transformation to polygonal ferrite in the heat-treated samples was suppressed.

The results seen in Table 3 corroborates the incomplete reaction phenomenon seen in bainitic steels, where, according to Garcia-Mateo et al. ${ }^{6}$ and Caballero et al. ${ }^{29}$, lower transformation temperatures should result in higher volumes of bainite. However, the obtained phase quantities are within the accuracy of the method of about \pm 3 wt.- $\%$, indicating that the effects on the phase contents are not pronounced. However, morphology and carbon content of the retained austenite is affected by the performed treatment, which is expected to influence the final mechanical properties, in particular the TRIP-behavior under load.

\section{General Discussion}

Granular bainite, which is the matrix of the material in its as-received condition, was characterized by Zajac et al. ${ }^{22}$ as an aggregate of irregular ferrite interwoven with islands of different morphologies and chemical compositions dependent of carbon partitioning during cooling. In the present work, ferrite is surrounded with MA, as shown in Figure 3c, because of the cementite precipitation suppression caused by silicon ${ }^{23}$.

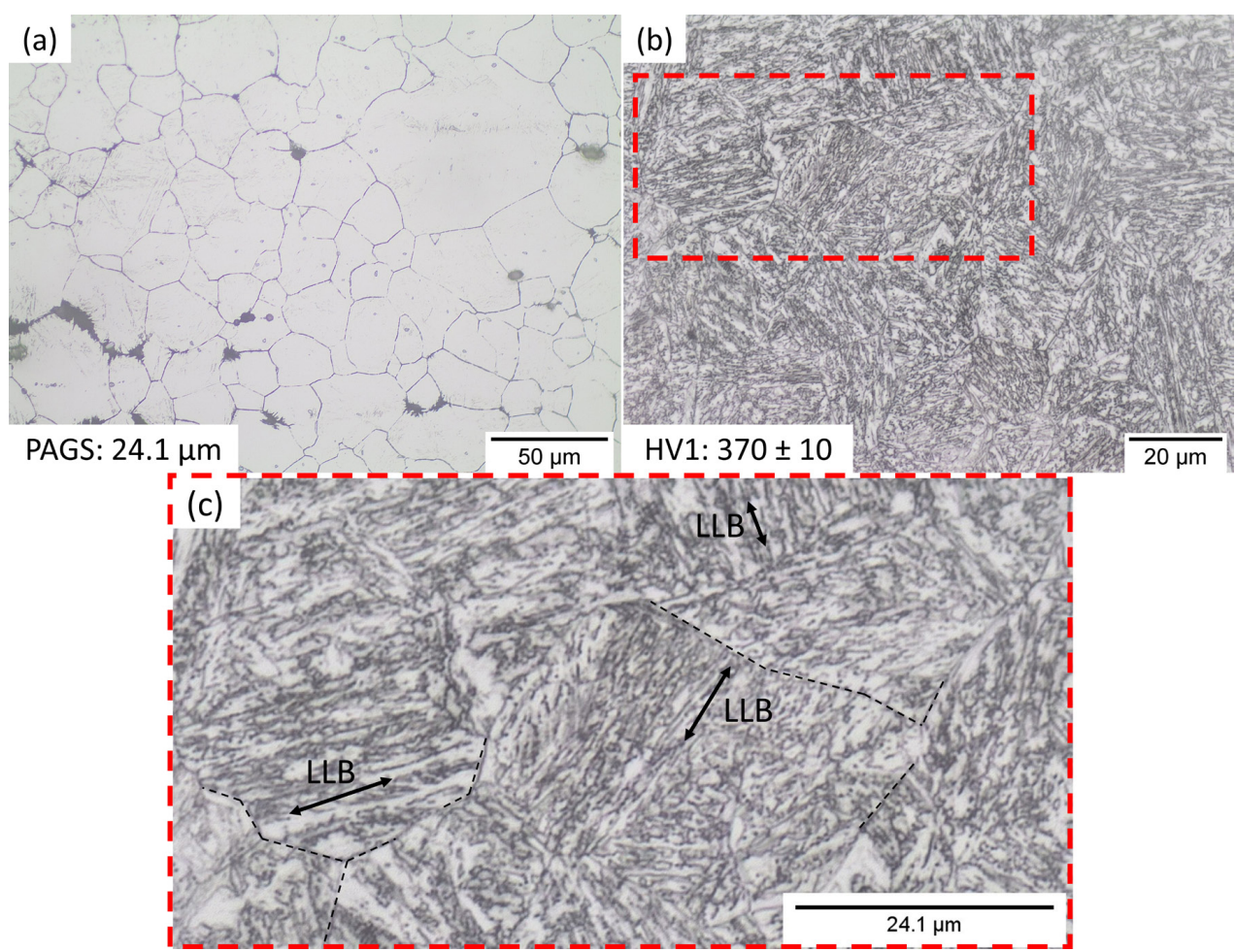

Figure 9. Core microstructures of the two-step heat treatment. (a) PAGS, (b) resulting microstructure and (c) magnification of delimited rectangle of (b). 


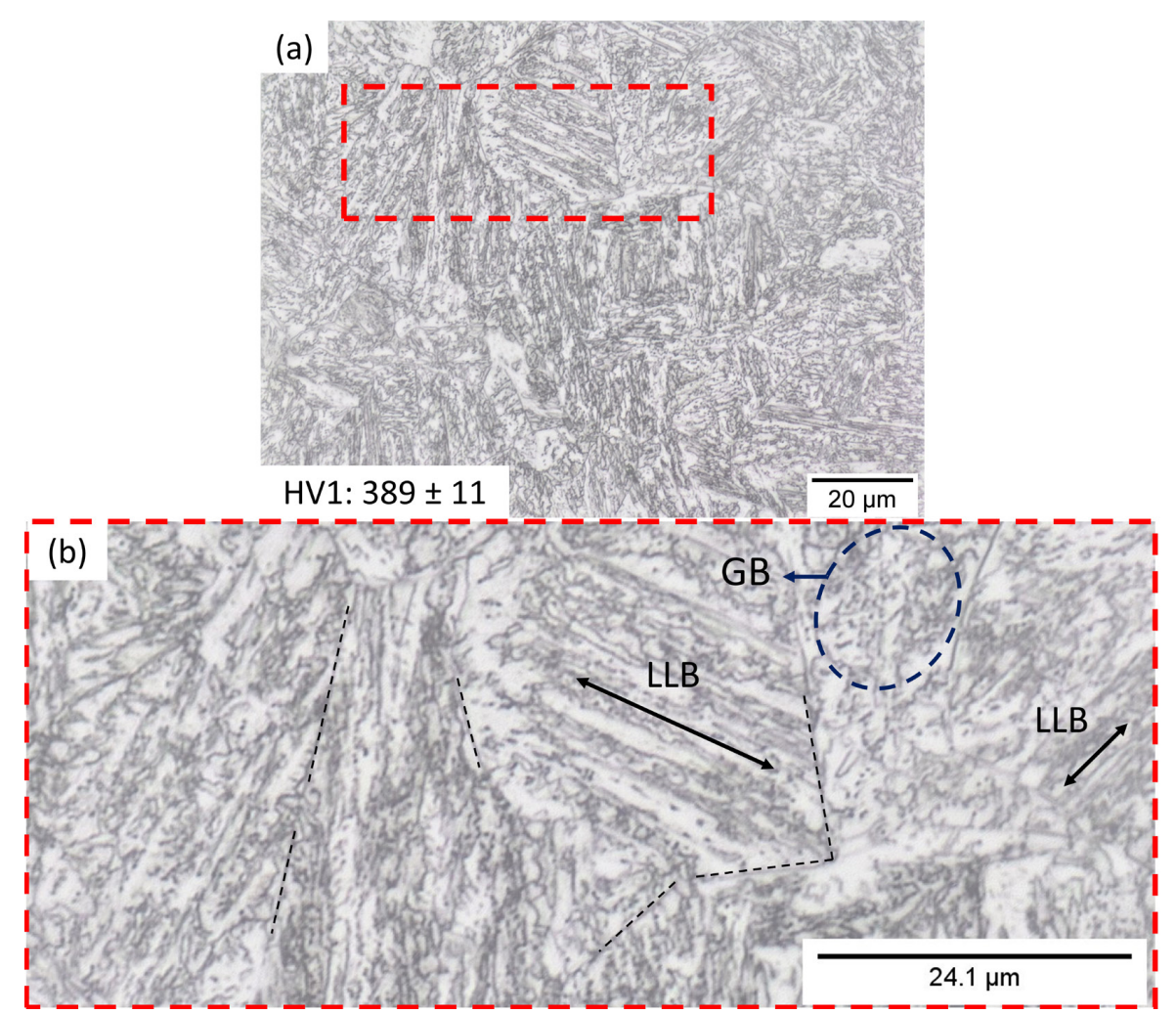

Figure 10. Middle radius microstructure of the two-step heat treatment. (a) resulting microstructure and (b) magnification of delimited rectangle of (a).

Table 3. Phase quantification obtained by Rietveld refinement.

\begin{tabular}{lcccc}
\hline \multirow{2}{*}{ Condition } & Region & $\begin{array}{c}\text { Austenite } \\
\text { content } \\
\text { (wt.-\%) }\end{array}$ & $\begin{array}{c}\text { Ferrite } \\
\text { content } \\
\text { (wt.-\%) }\end{array}$ & $\begin{array}{c}\mathrm{C}_{\mathrm{RA}} \\
\text { content } \\
\text { (wt.-\%) }\end{array}$ \\
\hline $\begin{array}{l}\text { Two-step heat } \\
\text { treatment }\end{array}$ & Core & 8 & 92 & 0.95 \\
\cline { 2 - 5 } As-received & $\begin{array}{l}\text { Madius } \\
\text { Middle }\end{array}$ & 8 & 92 & 0.9 \\
\hline
\end{tabular}

As seen by the conflicting views on GB growth modelling on many works ${ }^{21,30,31}$, there is still no consensus on the GB growth mechanism, since this microstructure presents both diffusional and adiffusional morphological evidences. As an example, this can be noticed by the partial maintenance of prior austenite grain boundaries. In Figure 3c, considering the average PAGS shown in the scale, it is noticeable that most of the prior boundaries have been consumed after the transformation, making way for a microstructure with a random granular aspect. However, this microstructure still exhibits regions with lath-like bainite and maintained PAGB, with MA morphology either accompanying the shape of grain boundaries or the bainitic laths, in concordance with what was proposed in the works of $\mathrm{Li}$ and $\mathrm{Baker}^{32}$ as well as Li et al. ${ }^{33}$. The microstructural duality of GB can be verified upon as the result of local driving force variation related to the inhomogeneous partitioning of carbon during continuous cooling transformation. This is similar to the dynamic partitioning effect seen in the work of Y.J Li et al. ${ }^{34}$. Besides that, the presence of polygonal ferrite, as found in Figure 3, is connected to the slow cooling used for the pre-process, which allows the reconstructive transformation from austenite to ferrite.

The effects of the two-step heat treatment, in a context of higher driving force for the bainitic transformation, conferred by the rapid cooling first step, resulted in an evident microstructural change that can be noticed between the heat-treated and as-received conditions. Primarily, the higher available driving force resulted in the formation of larger packets of lath-like bainite, which replaced the former granular bainite matrix, as shown in Figure 9 and Figure 10. Furthermore, the transformation to polygonal ferrite was avoided due to the diminished diffusion in the temperature range in which the transformation occurred.

Regarding the hardness variation between the as-received and heat-treated samples, there was an approximate increase of $55 \mathrm{HV}$ for the latter, as shown in Figure 3, Figure 9 and Figure 10. This can be explained by a coupled effect from the suppression of polygonal ferrite and the dislocation generation at the austenite/bainite interfaces. The key mechanism responsible for the change in the bainitic morphology and properties is connected to the mechanism of bainitic growth and the higher mechanical resistance of austenite in lower temperatures. As stated by J. Cornide et al. ${ }^{35}$, the displacive bainitic growth depends on the variation of lattice parameter from FCC to BCC, leading to necessary strain accommodations which arise in form of dislocations on the surrounding lattice. Dislocation build-up in the growing bainitic interface is also the cause of the growth cessation, because of the higher required driving force to surpass the increasing dislocated structures. This phenomenon of mechanical stabilization, is 
intensified as the temperature decreases, leading to thinner bainitic laths due to the hindrance of the interface development. He et al. ${ }^{4}$, connected the interface dislocation build-up and its influence on mechanical properties, supporting the present work hardness variation. On the other hand, in accordance with the works of Singh et al. ${ }^{36}$, and Caballero et al. ${ }^{37}$, the intrinsic higher austenite resistance at lower temperatures also acts as an interface motion suppressor contributing to lath refinement. At the optical microscopy scale, this was seen by the increased phase/constituent boundaries of the images, making the differentiation of the micro constituents more difficult. As a consequence of the diffusionless growth of lath-like bainite, there is no rearrangement of previous boundaries, which explains the maintenance of the PAGB in Figure $9 \mathrm{c}$ and Figure 10b. Although the obtained microstructure and increase in hardness supports the evidence of lath/ constituent refinement, it is not yet possible to completely assess the efficacy of the proposed two-step heat treatment in the tailoring of mechanical properties. It is expected that the microstructural refinement mainly improves the yield strength $^{4,38}$ and impact toughness ${ }^{39,40}$ while the higher content of austenite contributes to RA stability ${ }^{41,42}$ of the materials treated this way. However, tensile and impact tests were not in the scope of the present work.

Finally, comparing the core and middle radius regions, there was a slight increase in the hardness for the middle radius region. This, in agreement with Garcia-Mateo et al. ${ }^{6}$, is further evidence for lath refinement caused by the higher supercooling of this region, as provided by the simulation results (see section 3.4).

\section{Conclusions}

- A novel two-step continuous cooling heat treatment is proposed. It was designed and implemented on a low carbon bainitic steel. The heat treatment offers a promising path for microstructural optimization of continuous cooling bainitic steels at expense of a previous study on the cooling behavior of the part;

- The higher driving force supplied for the bainitic transformation allows higher volumes of lath-like bainite to form, replacing the blocky granular bainite matrix while avoiding reconstructive transformations;

- The growth mechanism difference of the investigated microstructures is reflected in the state of prior austenitic grain boundaries, hardness and phase/ constituent boundaries;

- As seen by the experimental and simulated obtained cooling rates, the designed heat treatment was reproducible.

\section{Acknowledgments}

This work was financially supported by CAPES (Program BRAGECRIM, process nr. 88881.142485/2017-01 - 1844/2017, PROEX program, process $88882.345785 / 2019-01)$ and CNPq (grant PQ 2018 process 308773-7) in Brazil and by the Deutsche Forschungsgemeinschaft (German Research Foundation - DFG) (via project EP 128/6-2 (project number 327887503 ) in Germany.

\section{References}

1. Buchmayr B. Critical assessment 22: bainitic forging steels. Mater Sci Technol (United Kingdom). 2016;32(6):517-22.

2. Sourmail T, Smanio V. Optimisation of the mechanical properties of air cooled bainitic steel components through tailoring of the transformation kinetics. Mater Sci Eng A. 2013;582:257-61.

3. Xu Z-B, Hui W-J, Wang Z-H, Zhang Y-J, Zhao X, Zhao X-M. Mechanical properties of a microalloyed bainitic steel after hot forging and tempering. J Iron Steel Res Int. 2017;24(11):1085-94.

4. He SH, He BB, Zhu KY, Huang MX. On the correlation among dislocation density, lath thickness and yield stress of bainite. Acta Mater. 2017;135:382-9.

5. Garcia-Mateo C, Caballero FG, Bhadeshia HKDH. Development of hard bainite. ISIJ Int. 2003;43(8):1238-43.

6. Garcia-Mateo C, Paul G, Somani M, Porter D, Bracke L, Latz A, et al. Transferring nanoscale bainite concept to lower C contents: a perspective. Metals (Basel). 2017;7(5):159.

7. Rementeria R, Jimenez JA, Allain SYP, Geandier G, Poplawsky JD, Guo W, et al. Quantitative assessment of carbon allocation anomalies in low temperature bainite. Acta Mater. 2017;133:333-45.

8. Gomez G, Pérez T, Bhadeshia HKDH. Air cooled bainitic steels for strong, seamless pipes Part 1 - alloy design, kinetics and microstructure. Mater Sci Technol. 2009;25(12):1501-7.

9. Sourmail T. Bainite and superbainite in long products and forged applications. HTM - Journal of Heat Treatment and Materials. 2017;72(6):371-8.

10. Wu H, Liu C, Zhao Z, Zhao Y, Zhu S, Liu Y, et al. Design of air-cooled bainitic microalloyed steel for a heavy truck front axle beam. Mater Des. 2006;27(8):651-6.

11. Wang XL, Wu KM, Hu F, Yu L, Wan XL. Multi-step isothermal bainitic transformation in medium-carbon steel. Scr Mater. 2014;74:56-9.

12. Avishan B, Tavakolian M, Yazdani S. Two-step austempering of high performance steel with nanoscale microstructure. Mater Sci Eng A. 2017;693:178-85.

13. Mousalou H, Yazdani S, Avishan B, Ahmadi NP, Chabok A, Pei Y. Microstructural and mechanical properties of low-carbon ultra-fine bainitic steel produced by multi-step austempering process. Mater Sci Eng A. 2018;734:329-37.

14. Li J, Liu F, Wang S, Li J, Liu Y, Meng Q. Effect of two-step bainite treatment on the morphology and texture of retained austenite and mechanical properties of austenitizing pretreated transformation-induced plasticity steel. Mater Sci Eng A. 2020;771:138567.

15. Dyson, D.J. Holmes B. Effect of alloying additions on the lattice parameter of austenite. J. Iron Steel Inst. 1970;208: 469-474.

16. Ericsson T. Principles of heat treating of steels. In: ASM Handbook Committee. ASM Handbook. Vol. 4: Heat Treating. Materials Park: ASM International; 1991. p. 14-30.

17. Silveira AC F, Bevilaqua WL, Dias VW, de Castro PJ, Epp J, Rocha AS. Influence of hot forging parameters on a low carbon continuous cooling bainitic steel microstructure. Metals (Basel). 2020;10(5):601.

18. Smoljan B. Numerical simulation of steel quenching. J Mater Eng Perform. 2002;11(1):75-9.

19. Guo Z, Saunders N, Schille JP. Modelling phase transformations and material properties critical to simulation of heat treatment distortion in steels. In: 17th IFHTSE Congress; 2008 Oct 27-30; Kobe, Japan. Proceedings. Japan: IFHTSE. p. 753-756.

20. Sugianto A, Narazaki M, Kogawara M, Shirayori A, Kim S-Y, Kubota S. Numerical simulation and experimental verification of carburizing-quenching process of $\mathrm{SCr} 420 \mathrm{H}$ steel helical gear. J Mater Process Technol. 2009;209(7):3597-609.

21. Qiao ZX, Liu YC, Yu LM, Gao ZM. Formation mechanism of granular bainite in a $30 \mathrm{CrNi} 3 \mathrm{MoV}$ steel. J Alloys Compd. 2009;475(1-2):560-4. 
22. Zajac S, Schwinn V, Tacke KH. Characterisation and quantification of complex bainitic microstructures in high and ultra-high strength linepipe steels. Mater Sci Forum. 2005;500-501:387-94.

23. Caballero FG. Carbide-free bainite in steels. In: Pereloma E, Edmonds DV, editors. Phase transformations in steels. Sawston, Cambridge: Woodhead Publishing; 2012. p. 436-67.

24. Reisinger S, Ressel G, Eck S, Marsoner S. Differentiation of grain orientation with corrosive and colour etching on a granular bainitic steel. Micron. 2017;99:67-73.

25. Wang X, Li B, Gu M. Simulation analysis on martempering in salt bath technology for carburized distortion sample. Metall Mater Trans, A Phys Metall Mater Sci. 2019;50(8):3758-66.

26. Narazaki M, Totten GE, Webster GM. Hardening by reheating and quenching. In: ASM Handbook Committee. Hanbook of residual stresses and deformation of steel. Materials Park: ASM International; 2002. p. 248-95.

27. Lan L, Chang Z, Fan P. Exploring the difference in bainite transformation with varying the prior austenite grain size in low carbon steel. Metals (Basel). 2018;8(12):988

28. Caballero FG, Chao J, Cornide J, García-Mateo C, Santofimia MJ, Capdevila C. Toughness deterioration in advanced high strength bainitic steels. Mater Sci Eng A. 2009;525(1-2):87-95.

29. Caballero FG, Bhadeshia HKDH, Mawella KJA, Jones DG, Brown P. Very strong low temperature bainite. Mater Sci Technol. 2002;18(3):279-84.

30. Caballero FG, Roelofs H, Hasler S, Capdevila C, Chao J, Cornide $\mathrm{J}$, et al. Influence of bainite morphology on impact toughness of continuously cooled cementite free bainitic steels. Mater Sci Technol. 2012;28(1):95-102.

31. Kang JS, Seol JB, Park CG. Three-dimensional characterization of bainitic microstructures in low-carbon high-strength lowalloy steel studied by electron backscatter diffraction. Mater Charact. 2013;79:110-21.

32. Li Y, Baker TN. Effect of morphology of martensite-austenite phase on fracture of weld heat affected zone in vanadium and niobium microalloyed steels. Mater Sci Technol. 2010;26(9):1029-40.
33. Li X, Ma X, Subramanian SV, Shang C, Misra RDK. Influence of prior austenite grain size on martensite-austenite constituent and toughness in the heat affected zone of 700MPa high strength linepipe steel. Mater Sci Eng A. 2014;616:141-7.

34. Li YJ, Kang J, Zhang WN, Liu D, Wang XH, Yuan G, et al. A novel phase transition behavior during dynamic partitioning and analysis of retained austenite in quenched and partitioned steels. Mater Sci Eng A. 2018;710:181-91.

35. Cornide J, Miyamoto G, Caballero FG, Furuhara T, Miller MK, García-Mateo C. Distribution of dislocations in nanostructured bainite. Diffus Defect Data Solid State Data Pt B Solid State Phenom. 2011;172-174:117-22.

36. Singh SB, Bhadeshia HKDH. Estimation of bainite plate-thickness in low-alloy steels. Mater Sci Eng A. 1998;245(1):72-9.

37. Caballero FG, Yen HW, Miller MK, Yang J-R, Cornide J, Garcia-Mateo C. Complementary use of transmission electron microscopy and atom probe tomography for the examination of plastic accommodation in nanocrystalline bainitic steels. Acta Mater. 2011;59(15):6117-23.

38. Zhu K, Bouaziz O, Oberbillig C, Huang M. An approach to define the effective lath size controlling yield strength of bainite. Mater Sci Eng A. 2010;527(24-25):6614-9.

39. Meng J, Feng Y, Zhou Q, Zhao L, Zhang F, Qian L. Effects of austempering temperature on strength, ductility and toughness of Low-C High-A1/Si carbide-free bainitic steel. J Mater Eng Perform. 2015;24(8):3068-76.

40. Zhou Y, Jia T, Zhang X, Liu Z, Misra RDK. Microstructure and toughness of the CGHAZ of an offshore platform steel. J Mater Process Technol. 2015;219:314-20.

41. Wang C, Ding H, Cai M, Rolfe B. Characterization of microstructures and tensile properties of TRIP-aided steels with different matrix microstructure. Mater Sci Eng A. 2014;610:65-75.

42. Guo H, Feng X, Zhao A, Li Q, Chai M. Effects of ausforming temperature on bainite transformation kinetics, microstructures and mechanical properties in ultra-fine bainitic steel. J Mater Res Technol. 2020;9(2):1593-605. 